\title{
JYD
}

http://jyd.pitt.edu/ | Vol. 12 Issue 1 DOI 10.5195/jyd.2017.486 | ISSN 2325-4017 (online)

\section{Beyond Evidence-Based Interventions for Teen Pregnancy Prevention}

\section{Emily Scribner-O'Pray}

Hennepin County

emily.scribner-opray@hennepin.us

\begin{abstract}
This article examines how the field of adolescent sexual health came to embrace evidence-based interventions (EBIs); whether or not this approach is effective in meeting the needs of adolescents, especially those at high risk for teen pregnancy; concerns related to the scaling up of EBIs; and identifies issues which must be resolved as we move forward.
\end{abstract}

\section{Introduction}

While evidence-based interventions (EBIs) have brought some important improvements to the field of adolescent sexual health, significant concerns must be addressed. As the field and funders have embraced EBIs as a way to improve outcomes and program efficacy we must be clear about the concerns related to and limitations of the adoption of EBIs as the primary method through which teen pregnancy prevention, sexuality education and adolescent reproductive health programs are provided.

To better understand these concerns, I interviewed experts in adolescent reproductive health and teen pregnancy prevention, gathered data from a focus group of youth workers who currently implement EBIs and drew from my own extensive experience in the adolescent reproductive health field and my experience as a program manager for a county-wide, evidence-based teen pregnancy prevention program in Minnesota. This article will examine how we came to embrace EBIs in the field of adolescent sexual health; whether or not this approach is effective in meeting the needs of adolescents, especially those at high risk for teen pregnancy; concerns related to the widespread implementation of EBIs; and identify issues which must be resolved as we move forward.

(cc) $\mathbf{E Y}$ New articles in this journal are licensed under a Creative Commons Attribution 4.0 License. This journal is published by the University Library System, University of Pittsburgh and is cosponsored by the University of Pittsburgh Press. The Journal of Youth Development is the official peer-reviewed publication of the National Association of Extension 4-H Agents and the National AfterSchool Association. 


\section{Beyond Evidence-Based Interventions-Teen Pregnancy}

"Evidence-based intervention" or "evidence-based practice" is a concept that started in the medical field and has increasingly become a focus in the fields of social work, chemical dependency, youth work, and adolescent health. An evidence-based intervention is "a prevention service (program, policy, or practice) that has been proven to positively change the problem being targeted. In general, there needs to be evidence that the intervention has been effective at achieving outcomes through some form of evaluation" (Baudry, 2013). Randomized control trials are typically considered the gold standard in ascertaining whether or not a program is "evidence-based."

\section{Background and History}

In the field of adolescent sexual health, EBIs evolved for a reason. Historically in the United States the topic of adolescent sexuality has been fraught with religious, moral, political and ideological controversy. In the wake of the HIV crisis in the late 1980s and early 1990s, and also due to the creation of the Adolescent Family Life Act in 1981 (Dailard, 2001), sex education in public schools became a political lightning rod. Conflicts erupted between those who believed that young people deserved comprehensive, medically accurate sexuality education and those that believed sex education should be left to families at home, or that abstinence-only-untilmarriage education was the only acceptable sex education for teenagers. Advocates for Youth defines abstinence-only programs as those that "teach abstinence as the only morally correct option of sexual expression for teenagers. They usually censor information about contraception and condoms for the prevention of sexually transmitted diseases and unintended pregnancy" (Abstinence-only-until-marriage programs, 2008). This battle was waged among politicians, school boards, policy makers and the public. Federal funding for abstinence-only education (which had existed since the 1980s as part of welfare reform) grew exponentially in the 1990s (Howell, 2007). It was argued by supporters of this funding that it was, in part to balance federal family planning funding, which provided contraceptive services to poor women of all ages. However no significant federal funding for comprehensive sexuality education existed.

In response to this situation, advocates for adolescent rights began to focus on "programs that work," with the argument that abstinence-only programs did not effectively delay the onset of sexual activity, or prevent teen pregnancies and sexually transmitted infections (STIs). At the time there was very little rigorous evaluation of sex education programs and there were no formalized structures for promoting evidence-based practice. Yet advocates for comprehensive sex education pointed to the fact that in many European countries sex education was wide- 
ranging and ongoing over the lifespan, family planning was readily accessible, and parents and children had more comfort with the topic of sexuality. In France, Germany and the Netherlands among other countries, teen pregnancy and STI rates were significantly lower than in the United States. In fact, the U.S. had (and still has) one of the highest teen birth rates of all developed nations (Alford \& Hauser, 2011).

Evaluation of sex education programs became a priority, and teen pregnancy and STIs, along with age of sexual debut (the age at which a young person first becomes sexually active) were outcomes that were most easily measured. While some experts in the field promoted what was referred to as a "youth development approach to pregnancy prevention," the focus was still on the prevention of teen pregnancy. Less concrete outcomes like healthy relationships, body image, comfort seeking sexual health services, positive sense of sexual identity, communication and negotiations skills, awareness of gender identity and roles were less easily measured, and were pushed to the side when it came to research. Even wider youth development outcomes of a sense of belonging, mastery, school success, leadership skills, positive contribution, connection to caring adults and a capacity to enjoy life were also not widely measured, though it is well known that these outcomes are interconnected with outcomes of pregnancy prevention (Gavin, Catalono, \& Markham, 2010). Preventing teen pregnancy was and still is a compelling argument for government investment (federal, state and local), as an economic argument could be made that preventing teen pregnancy saved taxpayers money.

Additionally, focusing on "programs that work" was a way that institutions, particularly public institutions, could avoid the controversy brought about by the idea of teens having sex, and provided justification for implementing programs that gave young people medically accurate, comprehensive information about sex, including topics like contraception, condoms, and access to reproductive health services. It gave schools and other institutions backing for decisions to include such controversial topics in health curriculums and youth programs. It was a way to ensure that young people had a right to access information and services to protect their health.

In 2010, the federal Department of Health and Human Services offered funding via the Office of Adolescent Health for programs to prevent teen pregnancy. Under tier I of the funding, $\$ 75$ million was allocated for the implementation of EBIs, and applicants could apply to implement one or two programs from a list of 28 that were evaluated and shown to be effective for teen pregnancy prevention. (Under tier II of the funding, another $\$ 25$ million was allocated for promising programs or to implement EBIs with a significant adaptation. All of tier II programs 


\section{Beyond Evidence-Based Interventions-Teen Pregnancy}

were required to include a randomized control trial.) This funding was important for truly putting EBIs on the map, as many smaller funders follow the lead of the federal government funding streams. Such a large source of funding solely for EBIs truly changed the landscape of adolescent sexual health programming.

\section{Do EBIs Work?}

Now, several years into the first large-scale funding for evidence-based teen pregnancy prevention programs, we should ask ourselves how this approach is working. There were clearly problems with the old model. Before the widespread use of EBIs, many organizations provided one-time classroom presentations which provided a positive conversation, a chance for clinics to have a "friendly face" in the community, and a chance for youth to have a neutral, knowledgeable adult to provide information, but not much chance for relationship building or youth involvement or youth development. There was also no evidence that these "one-shot" presentations effectively led to behavior change on the part of the adolescents who participated in them. Schools were haphazard at best with their approach to sex ed, often providing excellent sexuality education in one classroom and poor programming or even nothing in the class next door, with no consistency across districts or states. Youth-serving agencies ranged widely in their approach to working with young people around the topic of sexuality. Little evaluation was conducted, not for lack of interest, but for lack of resources and capacity. Evidence-based programs implemented with a plan to focus on programs that work and on populations most affected by the problems of teen pregnancy and STIs were an important step towards improving the situation.

It is right and reasonable that we expect that programs in general, and particularly those funded by public money, be effective. The use of EBIs has been a big step forward for the field in many ways. The implementation of programs that have been "proven to be effective" prevents controversy, encourages government investment, and as the data show, improves outcomes for young people in some very key areas. Youth workers like knowing that what they are doing "works" and that their efforts make a difference. Communities benefit from lower teen pregnancy rates and funding for programs that may not otherwise have been available.

However, implementing EBIs with strict fidelity to the program model poses its own challenges. Fidelity here means not changing anything from the original program, especially those program elements considered core components that contributed to the outcomes measured in the evaluation. We must not ignore these concerns and must continue to move forward in making 
sure that programs meet the needs of all youth and especially those who have the poorest outcomes.

\section{Concerns to Address}

One concern is the use of randomized control trials (RCTs) as the only acceptable option for evaluation when considering which programs are effective. RCTs are the gold standard for evaluation in order for a program to be considered an EBI. However, because RCTs are expensive and typically take place over several years, many programs deemed to be effective are decades old. Much has changed in the last 20 years. Technology, how teens relate to each other and the world, how they interact with schools and clinics, medical protocols and information, school environments and policies all affect how programs are integrated into young people's lives. Everything from No Child Left Behind to cell phones and the internet to how to treat pelvic inflammatory disease has vastly changed or is new since the time many of these programs were developed. As one focus group participant noted:

I think there's good stuff there and frameworks and good lessons.

There are also missing lessons, and some topics have really bad lessons. For example, everything in the curriculum says 'make a list'. That's not very interactive. Nothing has changed since the original curriculum was written in 1979. We need to modify scenarios to include technology, etc.

Another concern relates to the experience of young people who participate in EBIs. Much research on youth development has been conducted in recent decades, and there is a growing awareness of the way in which trauma affects young people's health status, education, decision-making and life trajectory. Many EBIs incorporate significant abstinence components, advocating abstinence as the "best choice" for young people and utilizing cautionary language about STIs and pregnancy to educate youth about potential harmful consequences of sexual activity. They emphasize that abstinence is the most responsible choice for those who have future goals, want to finish school and want to have successful careers. These messages may be effective for youth who are not yet sexually active, but could be off-putting or even harmful to young people who are currently sexually active, are teen parents, have a history of STIs, are GLBTQ, have been sexually trafficked or have experienced sexual trauma. Even the notion that sex is a choice a person can make and not one that is forced on them may be foreign to some adolescents. It must be noted that these same groups of young people are among those most at risk for the negative outcomes that adolescent sexual health programs seek to prevent. To 
this end, in 2015 the federal office funding the implementation of EBIs for teen-pregnancy prevention updated requirements, and require implementing agencies to review each program for a trauma-informed approach. Agencies are then required to address all identified concerns during implementation. Curricula are also required to be reviewed for positive youth development and LGBTQ inclusiveness. Whereas an ideal solution would be for curricula to be written from a trauma-informed, positive youth development and LGBTQ inclusive lens, this is a step in the right direction while newer curricula already written with these lenses are evaluated for effectiveness.

Although some evidence-based teen pregnancy prevention programs do incorporate youth engagement with the curriculum, implementing a program developed by someone in another part of the country with an entirely different group of youth in an entirely different decade and sticking with the original program curriculum makes it potentially difficult to engage youth authentically. Often these programs are delivered to youth and not with youth. Youth participants have little to no say in how the program is structured and in how, when, where and what learning happens. Some program models do incorporate a youth development framework in the EBI, like the Teen Outreach Program (TOP), which in addition to weekly lessons over nine months includes a service-learning component where youth identify a community need and respond to it by designing a service-learning project. Many programs however, offer a set number of educational sessions which must be offered in sequence and with little to no variation from the curriculum script. This leaves very little room for young people to truly engage the topics and ideas related to a very core part of their identity as human beings: their sexuality.

In addition to inhibiting youth voice, EBIs also can limit the autonomy, creativity and innovation of youth workers. One can assume that there are many things that "work" that we have not yet discovered. Yet if we do only what we already know works, we will not discover them. There must be a mechanism to experiment and learn new things, not only for our field, but to keep the youth worker engaged and effective. In focus groups, many youth workers identified that the repetitive nature of the EBI that they implement can become tedious. One youth worker noted, "The curriculum can be really repetitive. I like having a structure, but the youth can tell that I'm following a curriculum. I wish there was more flexibility in the order of lessons and how much was covered at once." We know that program effectiveness relies solidly on the effectiveness of the facilitator, and burn-out is a genuine concern. 
Many youth workers who are now implementing EBIs have already had education and work experience with a wider array of programming for youth, and draw on those skills and that knowledge to provide quality programming for youth. Everything that happens in a classroom doesn't always follow a script, and youth workers need to be able to respond effectively to questions, behavior and concerns that arise in the implementation of EBIs. One youth worker noted:

Part of following an EBI and making it effective is to know what's in the back of the patient's mind-if you haven't had a lot of previous experience working with youth with a program that allowed full flexibility, you may not really understand that and couldn't draw it out using the confines of the EBI.

In other words, this youth worker felt that her broader experience and training in sexuality education and youth development have been essential to truly understanding how youth think about sexuality and what concerns they may have. She feels that if she hadn't had the experience that allowed her to truly engage young people on their own terms around the topic of sex and sexuality, she would not be as effective working within the confines of the EBI. Another youth worker noted that "It would be difficult to do my EBI without the support of the team and without the background in sexual health."

EBIs also require a great deal of oversight, training and technical assistance to ensure that they are being implemented with fidelity to the program model and that the programs are high quality. Even with this oversight, it is nearly impossible to truly exactly replicate an EBI, as much of the adaptation is in response to barriers in any given setting. Efforts to replicate an evidence-based program in real-world settings bring into sharp focus the tension between adherence to the core elements of the program (fidelity) and adaptation in response to implementation challenges (Kelsey \& Layzer, 2014). Program oversight utilizes many resources by both the funder and the grantees. Licensing and training fees paid to program developers are often quite high and prohibitive for smaller agencies which may want to provide services related to adolescent sexual health. Agencies that have the capacity to manage large government grants can incorporate those costs into their larger grant applications, but for agencies that need to be more flexible and agile due to their small size and budgets this can be a barrier to the implementation of EBIs. This is particularly concerning when smaller funders follow suit with the federal government, requiring the implementation of EBIs, but don't offer funding that can cover the full cost of such implementation. 


\section{Considerations Moving Forward}

These problems are not unrecognized by agencies that fund EBIs. The Centers for Disease Control recently made a switch from advocacy for EBIs to advocacy for what they are calling "Exemplary Sexual Health Education" (ESHE), defined by the Centers for Disease Control as:

A systematic, evidence-informed approach to sexual health education that includes the use of grade-specific, evidence-based interventions, but also emphasizes sequential learning across elementary, middle, and high school grade levels. ESHE provides adolescents the essential knowledge and critical skills needed to avoid HIV, other STD, and unintended pregnancy. ESHE is delivered by well-qualified and trained teachers, uses strategies that are relevant and engaging, and consists of elements that are medically accurate, developmentally and culturally appropriate, and consistent with the scientific research on effective sexual health education. (Centers for Disease Control and Prevention, 2013)

It seems that this is an attempt to recognize that EBIs by themselves may not be adequate. The Office of Adolescent Health demonstrated their understanding of the need for program innovation by offering the tier II funding stream dedicated to promising approaches to teen pregnancy prevention. Additionally, the Office of Adolescent Health, which originally interpreted the idea of "fidelity" quite narrowly, allowing very few adaptations, seems to have learned over the course of the first round of funding that real-world implementation often makes adaptation necessary for programs to be feasible and responsive in a diverse array of environments. The Office of Adolescent Health seems to be focusing on an approach of allowing some adaptations to address barriers to implementation with consistent and ongoing oversight to ensure that core components of programs remain intact.

The field of adolescent health and its funders must begin to utilize other means of demonstrating effectiveness that do not rely solely on randomized control trials. While extremely valuable in validating our work, RCTs are expensive, require having a control group that does not receive services, take a long time to conduct and analyze, focus on problem prevention not positive youth development outcomes, and can lead to programs being outdated before they can be scaled up. Additionally, RCTs are often conducted by expert evaluators who have little if any experience as direct practitioners of youth work. Combining outcome-based 
evaluation with other forms of evaluation that include the voices and perspectives of youth and youth workers would be especially valuable. Additionally, expanding evaluation to include youth development outcomes is imperative if we truly want programs to encourage more holistic notions of sexual health.

We must also consider the people who implement EBIs. Programs are only as effective as the youth workers who facilitate them. Youth workers delivering EBIs must have access to support, training, technical assistance, opportunities for reflection and chances to participate in evaluation and have their perspectives and voices included. Having a community of other youth workers implementing similar programs was noted as particularly helpful by the focus group participants.

Finally, funding for the development, implementation and evaluation of innovative programs is necessary in order to ensure that the field continues to grow. Programs that are inclusive and responsive to diverse groups of youth; relevant to youth who have experienced trauma; youth who are marginalized and are most at risk for teen pregnancy are vital to ensure that we reach all young people. Programs must reflect the reality of the real world in which young people live today-and not the world in which they lived 20 years ago. This can happen only if there are mechanisms for innovation and creativity and young people are included in the development and evaluation of programs that serve them. Current EBIs must also continue to be reviewed and updated by program developers, youth workers and youth all taking part in assuring that programs are relevant.

\section{References}

Abstinence-only-until-marriage programs. (2008). Retrieved May 2014 from Advocates for Youth website: http://www.advocatesforyouth.org/topicsissues/abstinenceonly/757?task=view

Alford, S., \& Hauser, D. (2011, March). Adolescent sexual health in Europe and the US. Retrieved June 2014, from Advocates for Youth: http://www.advocatesforyouth.org/publications/publications-a-z/419-adolescent-sexualhealth-in-europe-and-the-us

Baudry, L. (2013, March). What is an evidence-based intervention (EBI)? Retrieved May 2014, from The Prevention Network website: 
http://www.preventionnetwork.org/Data/Sites/1/resourcesmaterials/evidencebasedinter ventions2013.pdf

Centers for Disease Control and Prevention. (2013, September 13). In Brief: Rationale for exemplary sexual health education (ESHE) for PS13-1308. (2013, September 13). Retrieved July 2014, from Centers for Disease Control, Adolescent and School Health website: http://www.cdc.gov/healthyyouth/fundedpartners/1308/strategies/education.htm

Dailard, C. (2001). Sex education: Politicians, parents, educators and teens. The Guttmacher Report on Public Policy, February 2001, 4(1), 9-12.

Gavin, L., Catalono, R., \& Markham, C. (2010). Positive youth development as a strategy to adolescent sexual and reproductive health. Journal of Adolescent Health, 46(3). 575591. doi: 10.1016/j.jadohealth.2009.11.215

Howell, M. (2007, July). The history of federal abstinence only funding. Retrieved June 30, 2014, from Advocates for Youth website: http://www.advocatesforyouth.org/publications/publications-a-z/429-the-history-offederal-abstinence-only-funding

Kelsey, M., \& Layzer, J. (2014). Implementing three evidence-based program models: Early lessons from the teen pregnancy prevention replication study. [Supplement]. Journal of Adolescent Health, 2014 March, 54, S45-S52. doi: 10.1016/j.jadohealth.2013.12.024 